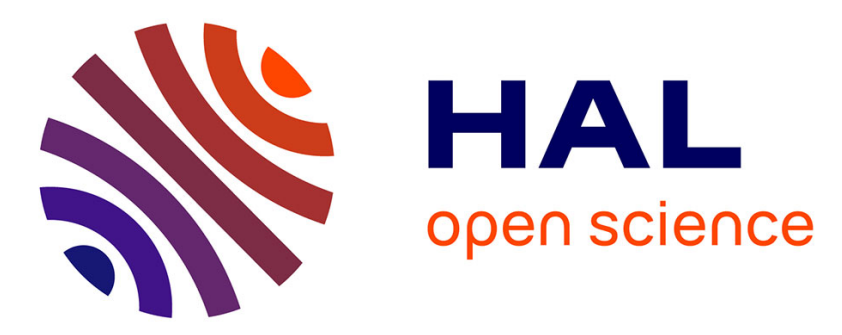

\title{
Perturbation of symmetric 3-RPR manipulators and asymptotic singularities
}

\author{
Michel Coste, Damien Chablat, Philippe Wenger
}

\section{To cite this version:}

Michel Coste, Damien Chablat, Philippe Wenger. Perturbation of symmetric 3-RPR manipulators and asymptotic singularities. F. Viadero, M. Ceccarelli. New Trends in Mechanism and Machine Science, Springer, pp.23-31, 2012, Mechanisms and Machine Science vol. 7, 978-94-007-4901-6. 10.1007/97894-007-4902-3 . hal-00674039

\section{HAL Id: hal-00674039 \\ https://hal.science/hal-00674039}

Submitted on 24 Feb 2012

HAL is a multi-disciplinary open access archive for the deposit and dissemination of scientific research documents, whether they are published or not. The documents may come from teaching and research institutions in France or abroad, or from public or private research centers.
L'archive ouverte pluridisciplinaire HAL, est destinée au dépôt et à la diffusion de documents scientifiques de niveau recherche, publiés ou non, émanant des établissements d'enseignement et de recherche français ou étrangers, des laboratoires publics ou privés. 


\title{
Perturbation of symmetric 3-RPR manipulators and asymptotic singularities
}

\author{
Michel Coste ${ }^{1}$, Damien Chablat ${ }^{2}$ and Philippe Wenger $^{3}$ \\ ${ }^{1}$ Université de Rennes 1, France, e-mail: michel.coste@univ-rennes1.fr \\ ${ }^{2}$ IRCCyN, Nantes, France, e-mail: damien.chablat@irccyn.ec-nantes.fr \\ ${ }^{3}$ IRCCyN, Nantes, France, e-mail: philippe.wenger@irccyn.ec-nantes.fr
}

\begin{abstract}
Small perturbations can affect the kinematic properties of manipulators with non-generic architecture. We study in this paper the perturbations of symmetric planar 3-RPR manipulators. We show in particular that one can obtain any of the three possible stable types of behaviour of 3-RPR manipulators for large values of the lengths of the legs.
\end{abstract}

Key words: Parallel manipulators, singularities, perturbation

\section{Introduction}

Planar parallel manipulators have been extensively studied in the past, see $[1,4,5$, $6,7,8,9,11,12]$ for example. Symmetric 3-RPR manipulators (those for which the platform triangle is congruent to the base triangle by an orientation-reversing isometry) have special kinematic properties (see [3]). Since these manipulators are not generic, the question arises of how a small perturbation of the architecture will influence the kinematic properties of the manipulator.

The configuration of asymptotic singularities of a symmetric 3-RPR manipulator is not stable. By "asymptotic singularities", we mean the limit of singularities (in the plane $r_{1}=$ constant, with coordinates $\left.\ell_{2}=r_{2}-r_{1}, \ell_{3}=r_{3}-r_{1}\right)$ as the length $r_{1}$ tends to infinity. For a generic 3-RPR manipulator, the curve of asymptotic singularities has two branches (connected components): an outer branch which is always an oval and an inner one, with three possible stable types (diamond, swallowtail and annulus following the terminology of [2] - see Figure 3). The asymptotic direct kinematic problem has at most four solutions. In the case of a symmetric 3-RPR manipulator, the inner branch of the curve of asymptotic singularities is reduced to a point. We show that any of the three possible stable types can be obtained by a small perturbation of a symmetric 3-RPR manipulator.

The curve of singularities of a symmetric 3-RPR manipulator, in the plane $r_{1}=$ constant with coordinates $r_{2}^{2}-r_{1}^{2}, r_{3}^{2}-r_{1}^{2}$, has an inner branch independent of the length $r_{1}$ if it is large enough. This branch is a deltoid with three cusps (cf 
[3]). The image of this deltoid in the plane with coordinates $\left(\ell_{2}, \ell_{3}\right)$ tends to the point $(0,0)$ (the degenerate inner branch of the asymptotic singularity curve) as $r_{1}$ tends to infinity. The direct kinematic problem has 6 solutions inside this deltoid and 2 outside. The singularity surface in the space with coordinates $\left(r_{1}^{2}, r_{2}^{2}, r_{3}^{2}\right)$ has a component which is a portion of a cylinder with base this deltoid. Actually, the inverse kinematic mapping induces a two-to-one map of a component of the singularity surface in the workspace to this cylinder; so the deltoid is to be considered as a double curve. A small perturbation will deform the double deltoid to a closed curve with 6 cusps and a few self-intersections. We mean that we shall obtain such a curve as the inner branch of the section $r_{1}=$ constant of the singularity surface in the $\left(r_{1}, r_{2}, r_{3}\right)$ space, for some sufficiently large value of $r_{1}$ and a small perturbation of the architecture. We study examples of the transition from this perturbed double deltoid with 6 cusps, as the length $r_{1}$ increases, to the stable asymptotic type with 4 or 0 cusps.

\section{Asymptotic singularities}

We recall a few results from [2] with some extra comments. The notations we use are made clear in Figure 1. Here, $b_{A}, h_{A}, b_{B}, h_{B}$ are all positive. The parameters $d_{A}$ and $d_{B}$ may have any real value. The triangle $B_{1} B_{2} B_{3}$ may be oriented counter-clockwise (as in Figure 1) or clockwise.

Fig. 1 Notations and parameters for the 3-RPR manipulator

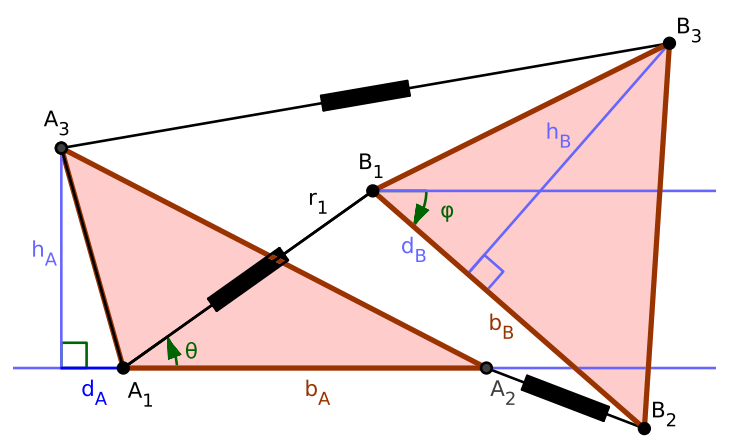

We have defined the notion of asymptotic singularities in the introduction and mentioned the fact that they consist of two branches. From a geometric viewpoint, asymptotic singularities are characterized by the fact that there exists $k \in \mathbb{R}$ such that

$$
\overrightarrow{p\left(B_{1}\right) p\left(B_{2}\right)}=k \overrightarrow{p\left(A_{1}\right) p\left(A_{2}\right)} \quad \text { and } \quad \overrightarrow{p\left(B_{1}\right) p\left(B_{3}\right)}=k \overrightarrow{p\left(A_{1}\right) p\left(A_{3}\right)},
$$


where $p$ is a projection parallel to the direction given by $\theta$, which is the common direction of the infinite legs (see Figure 2).

Fig. 2 Asymptotic singular configuration (with $k=-0.45$ )
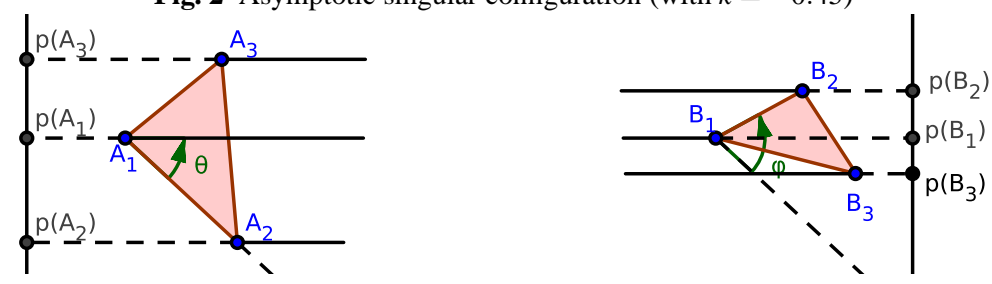

If $\theta$ is fixed, the condition (1) is realized for two values of the angle $\varphi$ which differ by $\pi$, corresponding to the cases $k>0$ and $k<0$. The case $k<0$ (resp. $k>0$ ) is the case of singularities with "crossed (resp. non-crossed) legs". The inner branch of the asymptotic singularity curves always contains the image of $\theta=0, \varphi=0$. If the base and platform triangles have reverse orientations, the inner branch is the branch of singularities with crossed legs (as in Figure 2); if both triangles have the counter-clockwise orientation, the inner branch is the branch of singularities with non-crossed legs.

It was proved in [2] that, for a generic 3-RPR manipulator, there are only three possible types for the curve of asymptotic singularities: diamond, swallowtail and annulus. These three types are depicted in Figure 3

Fig. 3 Diamond, swallowtail and annulus

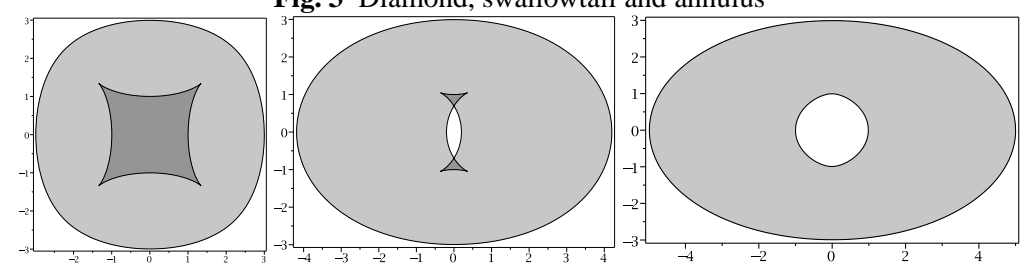

In the three examples of Figure 3, we have $b_{A}=2, h_{A}=1, d_{A}=d_{B}=0, h_{B}=2$. The leftmost type (diamond) is obtained for $b_{B}=1$, the one in the middle (swallowtail) for $b_{B}=2.2$ and the rightmost one (annulus) for $b_{B}=3$. In each picture, the light gray indicates two solutions for the direct kinematic problem DKP), and the medium gray four.

The quantities which discriminate between the three stable asymptotic configurations of asymptotic singularities are

$$
Q_{1}=U_{A, B}-\left(S_{A}^{2}+S_{B}^{2}\right) \quad Q_{2}=U_{A, B}^{3}-S_{A}^{2} S_{B}^{2}\left(3 U_{A, B}+S_{A}^{2}+S_{B}^{2}\right)
$$


where $S_{A}=b_{A} h_{A} / 2$ and $S_{B}=b_{B} h_{B} / 2$ are the areas of the triangles and

$$
U_{A, B}=\left(b_{A}^{2} h_{B}^{2}+h_{A}^{2} b_{B}^{2}+\left(b_{A} d_{B}-d_{A} b_{B}\right)^{2}\right) / 4 .
$$

The configuration of asymptotic singularities is a diamond when $Q_{1}>0$, a swallowtail when $Q_{1}<0$ and $Q_{2}>0$, an annulus when $Q_{2}<0$; it is impossible to have $Q_{1}>0$ and $Q_{2}<0$ (cf. [2]).

\section{Asymptotic singularities and kinematic properties of the manipulator}

The stability of asymptotic types means that they are unchanged by small perturbations of the architecture of the manipulator. But it also means that asymptotic singularities give information on kinematic properties of the manipulator for sufficiently large values of the length of legs. More precisely, if a 3-RPR manipulator has one of the stable types of asymptotic singularities, then the sections of the singularity surface by the planes $r_{1}=$ constant will all have the same type for sufficiently large values of $r_{1}$. We illustrate this property with the example for the diamond type: $b_{A}=2, h_{A}=1, d_{A}=0, b_{B}=1, h_{B}=2, d_{B}=0$. There are two cases to consider: the case when both triangles have the same orientation and the case when the orientations are opposite.

When both triangles are oriented likewise, the section of the singularity surface has the diamond type already when $r_{1}=3$, as shown in Figure 4 . Note that the inner branch of the singularity curve (singularities with non-crossed legs) is almost coincident with the inner branch of the asymptotic curve (dotted line).

Fig. 4 Diamond type, base and platform with same orientation

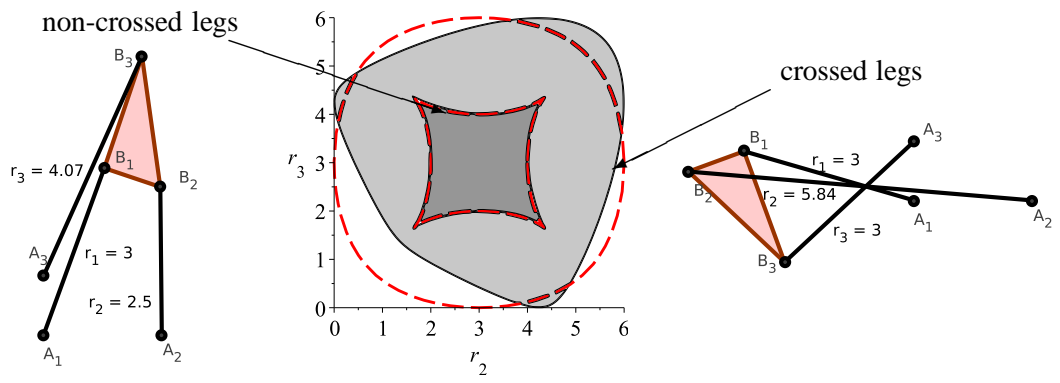

When the triangles have opposite orientations, the asymptotic type is attained for larger values of $r_{1}$. Figure 5 shows the section for $r_{1}=5$. Note that it is now the outer branch (still the singularities with non-crossed legs) which is almost coincident with its asymptotic counterpart. 
Fig. 5 Diamond type, base and platform with reverse orientations

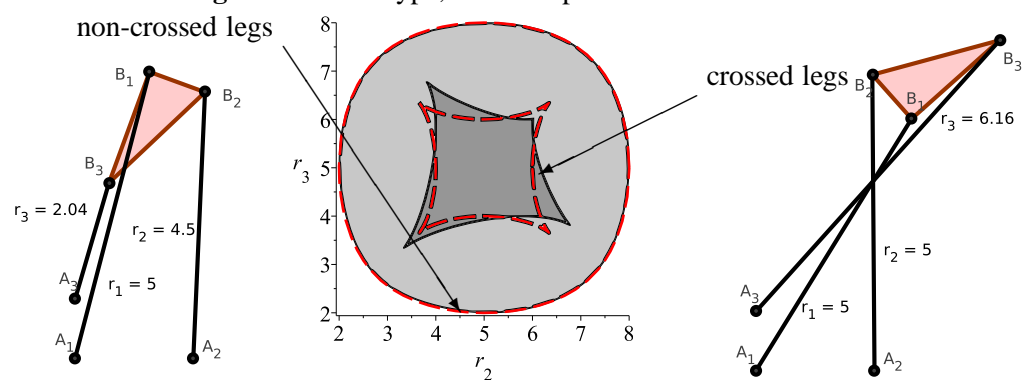

\section{Perturbation of symmetric manipulator}

The parameters of a symmetric manipulator satisfy $b_{B}=b_{A}, h_{B}=h_{A}$ and $d_{B}=d_{A}$. In this case both quantities $Q_{1}$ and $Q_{2}$ vanish; this is an indication for the fact that any of the three stable types may be obtained by a perturbation of a symmetric manipulator. Reciprocally, if $Q_{1}=Q_{2}=0$ then one must have $b_{B}=b_{A}, h_{B}=h_{A}$ and $d_{B}=d_{A}$, which means that either we have a symmetric manipulator or a manipulator whose base and platform triangles are congruent via an orientation-preserving isometry.

We perturb a symmetric 3-RPR manipulator whose base and platform are right triangles $A_{1} A_{2} A_{3}$ and $B_{1} B_{2} B_{3}$, so that $d_{A}=d_{B}=0$. We perturb it keeping $d_{A}=d_{B}=$ 0 . Carrying the values for $d_{A}, d_{B}$ in the expressions (2) for $Q_{1}$ and $Q_{2}$, we obtain

$$
\begin{aligned}
Q_{1}^{\text {pert }} & =\frac{\left(b_{A}-b_{B}\right)\left(b_{A}+b_{B}\right)\left(h_{B}-h_{A}\right)\left(h_{B}+h_{A}\right)}{4} \\
Q_{2}^{\text {pert }} & =\frac{\left(b_{A}^{2} h_{B}-h_{A} b_{B}^{2}\right)\left(b_{A}^{2} h_{B}+h_{A} b_{B}^{2}\right)\left(b_{A} h_{B}^{2}-h_{A}^{2} b_{B}\right)\left(b_{A} h_{B}^{2}+h_{A}^{2} b_{B}\right)}{64}
\end{aligned}
$$

Fix $b_{A}$ and $h_{A}$ (for instance $b_{A}=2, h_{A}=1$ ) and let $b_{B}$ and $h_{B}$ vary in the positive quadrant. This quadrant is cut by the two half-lines $b_{B}=b_{A}, h_{B}=h_{A}$ and the two half-parabolas $h_{B}=h_{A} b_{B}^{2} / b_{A}^{2}, b_{B}=b_{A} h_{B}^{2} / h_{A}^{2}$ into regions labelled $\mathrm{Di}$, Sw or An according to the asymptotic type (see Figure 6 ).

All regions are adjacent to the point $b_{B}=b_{A}, h_{B}=h_{A}$ corresponding to the symmetric manipulator. This shows that a symmetric manipulator can be perturbed to any of the stable asymptotic types. One can also spot on Figure 6 the three points $(1,2),(2.2,2)$ and $(3,2)$ corresponding to the examples in Figure 3.

Note that the description of perturbation concerning asymptotic singularities also applies to the non generic manipulator with two congruent triangles both with counter-clockwise orientation. 
Fig. 6 Stable types of perturbations of a symmetric manipulator

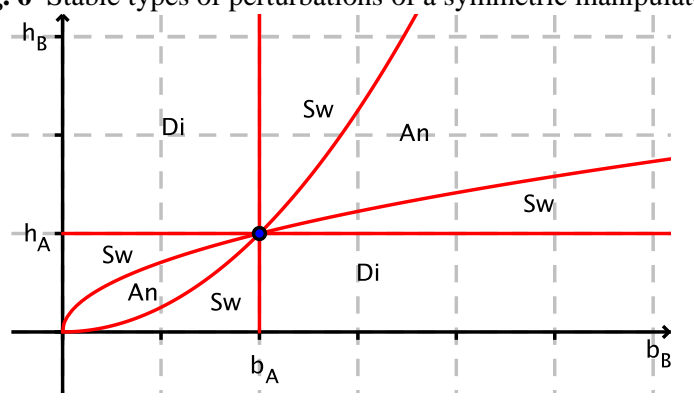

\section{Transition to the asymptotic configuration}

We now examine in detail the effect of a perturbation of the geometry of a symmetric manipulator on its kinematic properties. We start with a symmetric manipulator whose base and platform are isosceles right triangles of side $1\left(b_{A}=b_{B}=h_{A}=\right.$ $\left.h_{B}=1, d_{A}=d_{B}=0\right)$. We focus on the inner branch of the curve of singularities in the $\left(r_{2}, r_{3}\right)$ plane at $r_{1}=$ constant (with $r_{1} \geq 3$ ). Indeed, nothing interesting happens for the outer branch which is an oval delimiting regions where the DKP has 0 or 2 solutions. The inner branch is a deltoid with three cusps; the DKP has 6 solutions inside the deltoid and 2 outside.

Fig. 7 Symmetric manipulator: slices at $r_{1}=3,10,20$
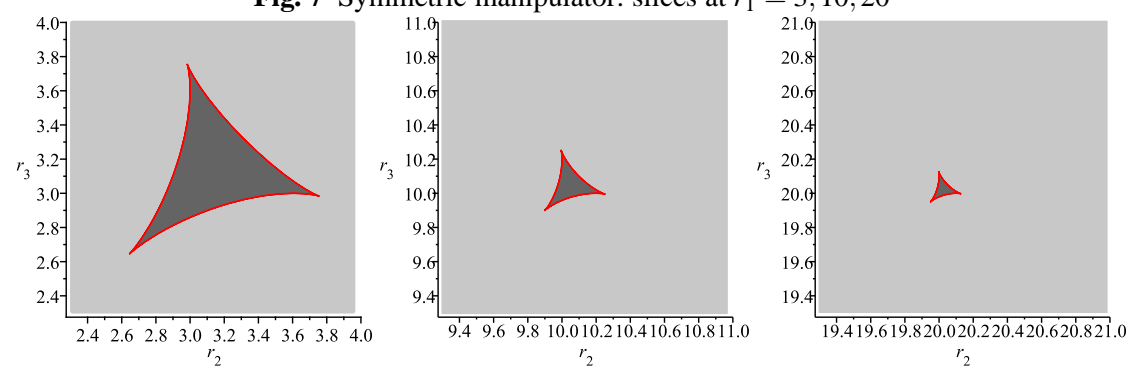

Figure 7 shows the slices at $r_{1}=3,10,20$. We use here the same gray scale code as above: light gray for 2 solutions to the DKP, medium gray for 4 and dark gray for 6 (only 2 and 6 in Figure 7). The deltoid is always here. If it were drawn with coordinates $\left(r_{2}^{2}, r_{3}^{2}\right)$, it will be independent of $r_{1}$ up to translation. But here, with $\left(r_{2}, r_{3}\right)$ coordinates, it shrinks to a point as $r_{1}$ tends to infinity.

We now perturb the geometry of the platform by taking $b_{B}=1.2, h_{B}=0.8$ and $d_{B}=0$. This is a rather large perturbation, so that its effect is well visible; from the analysis of the preceding section, we know that the perturbed manipulator will 
have a diamond asymptotic configuration. Figure 8 shows again sections at $r_{1}=$ $3,10,20$. The picture on the left exhibits a perturbed double deltoid, a closed curve with six cusps and three self-intersections; the next pictures show how it evolves to a diamond with the vanishing of a pair of cusps. The inner branch stabilizes (up to translation) as $r_{1}$ tends to infinity; one can check that the size of the stabilized picture is approximately linear in the amplitude of the perturbation.

Fig. 8 Perturbation to diamond: slices at $r_{1}=3,10,20$

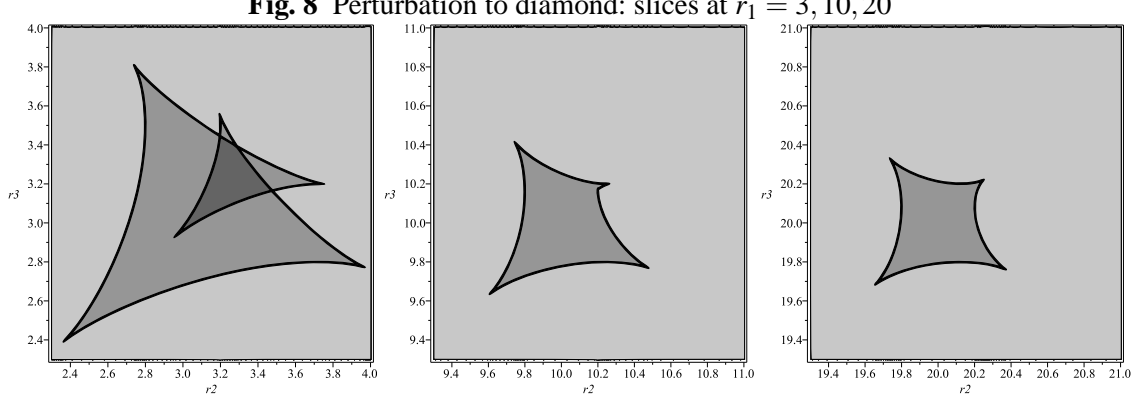

A similar analysis can be done for perturbations to the other stable asymptotic configurations. Taking $b_{B}=1.2, h_{B}=1.05$ and $d_{B}=0$ we obtain a perturbation to swallowtail (Figure 9, first row); taking $b_{B}=1.2, h_{B}=1.2$ and $d_{B}=0$ we obtain a perturbation to annulus (Figure 9, second row). In both cases an inner region with no solution of the DKP appears; all cusps disappear in the annulus case, thus ruling out the possibility of non-singular assembly mode change for large values of $r_{1}$ (see [12]). The slices in Figure 9 are taken for $r_{1}=3,10,30$.

\section{Conclusion}

We have shown how a perturbation of the geometry of a symmetric 3-RPR manipulator affects its kinematic properties. In particular, we proved that these manipulators are at the bifurcation point between the three different types of stable asymptotic kinematic behavior for 3-RPR manipulators. The point in studying the effect of perturbations on the kinematic properties is that, in the real world, one cannot achieve with certainty the specifications of non-generic architectures (such as the symmetry condition in our study).

\section{References}

1. Bonev, I., Zlatanov,D. and Gosselin, C.: Singularity analysis of 3-DOF planar parallel mech- 
Fig. 9 Perturbations to swallowtail and to annulus: slices at $r_{1}=3,10,30$

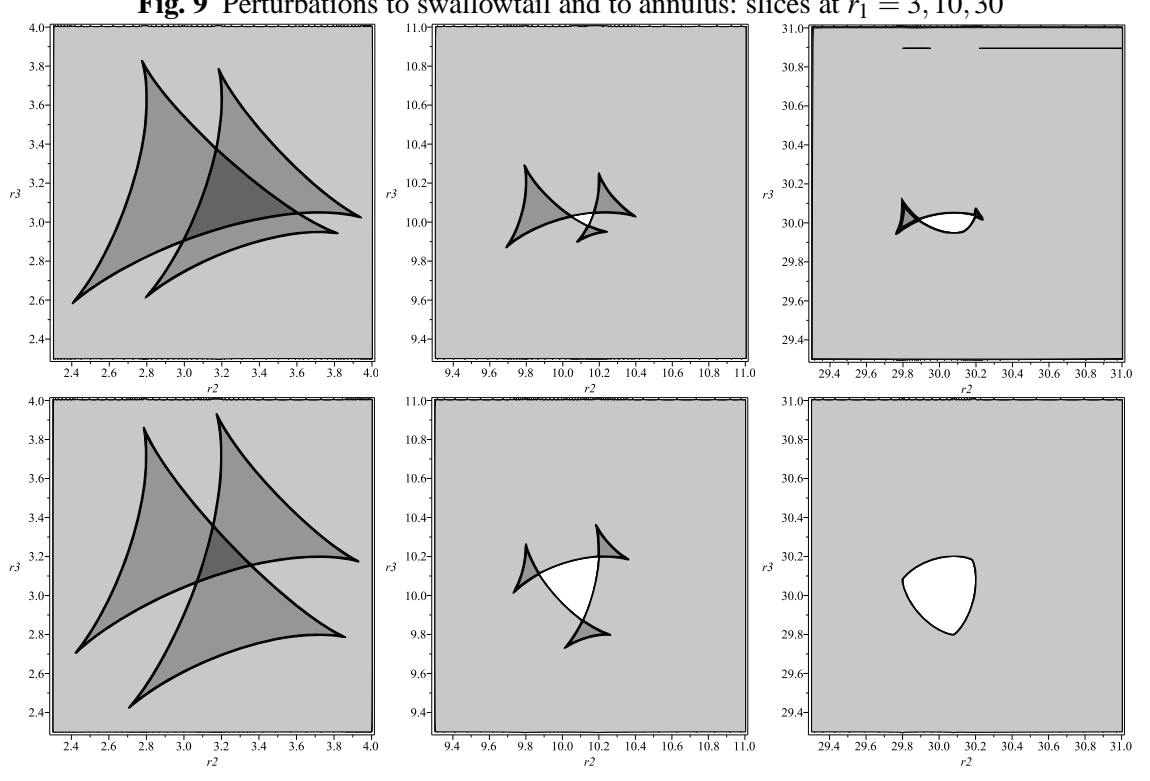

anisms via screw theory. ASME Journal of Mechanical Design, 125(3), 573-581 (2003)

2. Coste, M.: Asymptotic singularities of planar parallel 3-RPR manipulators, preprint (2012) http://hal.archives-ouvertes.fr/docs/00/65/64/60/PDF/asymp.v3.pdf

3. Coste, M., Wenger, P. and Chablat, D.: Singular surfaces and cusps in symmetric planar 3RPR manipulators. In: Proc. of IEEE/RSJ International Conference on Intelligent Robots and Systems IROS 2011, San Francisco, pp.1453-1458 (2011)

4. Gosselin, C.M. and Merlet, J-P.: On the direct kinematics of planar parallel manipulators: special architectures and number of solutions. Mechanism and Machine Theory, 29(8), 10831097 (1994)

5. Hunt, K.H.: Structural kinematics of in-parallel actuated robot arms. J. of Mechanisms, Trans. and Aut. in Design 105(4), 705-712 (1983)

6. Husty, M.L.: Non-singular assembly mode change in 3-RPR-parallel manipulators. In: Computational Kinematics: Proceedings of the 5th International Workshop on Computational Kinematics, Springer Verlag, pp.51-60 (2009)

7. Innocenti, C. and Parenti-Castelli, V.: Singularity-free evolution from one configuration to another in serial and fully-parallel manipulators. In: Proc. ASME Design Technical Conferences, DE-Vol. 45, Spatial mechanisms and mechanical systems, pp.553-560 (1992)

8. Mcaree, P.R. and Daniel, R.W.: An explanation of never-special assembly changing motions for 3-3 parallel manipulators. The International Journal of Robotics Research, 18(6), 556-574 (1999)

9. Merlet, J-P.: Parallel Robots (Series: Solid Mechanics and Its Applications). Springer (2006)

10. Wenger, P. and Chablat, D.: Uniqueness domains in the workspace of parallel manipulators. In: I.F.A.C-Symposium on Robot Control (SYROCO'97), Nantes, pp.431-436 (1997)

11. Wenger, P. and Chablat, D.: Workspace and assembly-modes in fully-parallel manipulators: a descriptive study. In Advances in Robot Kinematics and Computational Geometry, Kluwer Academic Publishers, pp. 117-126 (1998)

12. Zein, M., Wenger, P. and Chablat, D.: Singular curves in the joint space and cusp points of 3-RPR parallel manipulators. Robotica, 25(6), 717-724 (2007) 